\title{
Study of silver films over silica beads as \\ a surface-enhanced Raman scattering (SERS) substrate for detection of benzoic acid
}

\author{
Li-Li Bao, Shannon M. Mahurin, Cheng-Du Liang and Sheng Dai*
}

Chemical Sciences Division, Oak Ridge National Laboratory, Oak Ridge, Tennessee 37831, USA

Received 2 October 2003; Accepted 15 January 2003

\begin{abstract}
Surfaces prepared by vacuum depositing silver on silica nanospheres were investigated as surfaceenhanced Raman scattering (SERS) substrates using benzoic acid as an analyte. The SERS sensitivity of the substrates was probed as a function of substrate parameters, such as silver-film thickness and silica-bead dimensions. The SERS signals were found to increase with an increase in silver-film thickness and silica-bead diameter until a maximum signal was reached. Additional increases in the film thickness or the bead diameter resulted in a decrease in the SERS signal. Maximum SERS response was obtained with a substrate made by vapor depositing a $6 \mathrm{~nm}$ thick coating of silver on a thin layer of silica beads with a nominal diameter of $565 \mathrm{~nm}$. Scanning electron microscopy (SEM) of the substrates was performed to consider the effect of surface morphology on the SERS responses. Optimal SERS substrates exhibited a detection limit of $5 \times 10^{-7} \mathrm{M}$ for adsorbed benzoic acid. A linear relationship was demonstrated between the SERS response and the concentration of benzoic acid with two different slopes, one in the $10^{-5}-10^{-3} \mathrm{M}$ range and the other in the $10^{-3}-10^{-1} \mathrm{M}$ range. Published in 2003 by John Wiley \& Sons, Ltd.
\end{abstract}

KEYWORDS: silver films; silica beads; surface-enhanced Raman scattering; benzoic acid

\section{INTRODUCTION}

Surface-enhanced Raman scattering (SERS) has long been applied as a convenient and sensitive technique to investigate surface adsorbates. ${ }^{1}$ Among the useful substrates for SERS measurements, silver is the most commonly used. Electrochemically roughened silver electrodes were the first surfaces to produce enhanced Raman signals. ${ }^{2}$ Since that initial discovery, a number of methods have been explored to prepare SERS-active substrates of silver. One approach that has become popular is the colloidal dispersion of metal particles. ${ }^{3}$ Freeman et al. reported highly active surfaces synthesized by self-assembly of gold and silver colloid monolayers on polymer-coated substrates. ${ }^{4}$ Another commonly used method for preparing active substrates is the vapor deposition of metal films. ${ }^{5-7}$ Vapor deposition offers the advantage of some control over the morphology and optical properties of the film which directly affect the sensitivity of the SERS surface. Moody et al. developed a novel SERS

*Correspondence to: Sheng Dai, Chemical Sciences Division, Oak Ridge National Laboratory, Oak Ridge, Tennessee 37831, USA. E-mail: dais@ornl.gov

Contract/grant sponsor: US Department of Energy; Contract/grant number: DE-AC05-00OR22725. substrate based on vapor deposition of silver on polystyrene microspheres. ${ }^{8}$ The effects of experimental parameters (e.g. sphere size, silver thickness, and excitation wavelength) on SERS responses were investigated. ${ }^{8}$ An early experiment demonstrated a relatively simple procedure for producing SERS-active substrates in which the silver film was deposited by Tollens reaction on to frosted glass microscope slides. ${ }^{9}$ Although fairly easy to prepare, these films are typically less sensitive than other films. The most straightforward method for generating SERS-active substrates is by nitric acid etching of metal foils. ${ }^{10}$ Silver-doped sol-gel films coated on glass substrates have also been used as SERS surfaces. Films prepared by the sol-gel processing method exhibit good long-term stability and sensitivity. ${ }^{11,12}$

For solid metal films, the efficiency of the substrates is often influenced by the lack of stability of the metal surfaces. The stability of these surfaces can be significantly improved by the addition of a silica support for the silver. Silica has often been used to support catalysts. Introduction of the silica into the system uniformly disperses the silver and enhances the stability of the surface by preserving the aggregation of the silver particles. ${ }^{13}$ Numerous methods have been utilized to introduce the stabilizing silica into the silver films. For example, Liu et al. prepared substrates by 
impregnating silica with silver ions in the form of $\mathrm{Ag}\left(\mathrm{NH}_{3}\right)_{2}^{+}$ followed by calcination to reduce the silver ions to metallic silver. ${ }^{13}$ Silica spheres containing homogeneously dispersed silver quantum dots prepared by photochemical reduction of silver ions in a microemulsion reaction matrix during the hydrolysis of tetraethyl orthosilicate (TEOS) have also been used as SERS substrates. ${ }^{14}$

Much research effort has lately been focused on developing substrates with silver nanoparticles or metal islands in a silica host. In particular, silver nanoparticles embedded into silica thin films have been developed by thermal reduction of silver organometallic compounds. ${ }^{15}$ Silver nanoparticles have been prepared on silica spheres by pretreatment steps in electroless plating ${ }^{16}$ in which oxidation of the $\mathrm{Sn}^{2+}$ modified on the silica sphere and the simultaneous reduction of the silver ions in solution was utilized and the resulting metallic silver was attached to the silica surface in the form of nanoparticles. Silver films deposited on silica nanosphere surfaces have been prepared by the method of vacuum evaporation to produce SERS active films suitable for use in ultrahigh vacuum. By combining temperature-programmed desorption and SERS substrates made by vacuum deposition of silver on silica nanospheres, surface chemistry and adsorption energetics have been probed in detail in an ultrahigh vacuum environment. ${ }^{17}$ Among the silica-supported silver substrates, those prepared by vacuum deposition have the advantage that the surface roughness is extremely reproducible and temporally stable for many days (when protected from atmospheric surface contamination). ${ }^{18}$

In this work, silver-coated silica spheres were investigated as highly SERS-active substrates. The effect of substrate parameters such as silver thickness and silica bead size on SERS sensitivity was studied. Observations regarding the surface morphology of the substrates with respect to the SERS sensitivity are presented. The sensitivity of the substrates was analyzed in detail by observing the intensity of SERS peaks from benzoic acid. The SERS response was found initially to increase as the thickness of the silver film and the diameter of the silica beads were increased. Maximum SERS response was produced by a film composed of $565 \mathrm{~nm}$ silica beads coated with a $6 \mathrm{~nm}$ layer of silver.

\section{EXPERIMENTAL}

\section{Materials}

Tetraethyl orthosilicate (TEOS) and methanol were obtained from Aldrich, ethanol (absolute) from AAPER Alcohol and Chemical Co. and ammonia solution (28-30\%) from J. T. Baker. All chemicals were used without further purification. Various concentrations of benzoic acid (Aldrich) in methanol were obtained by serial dilution of a stock solution. Glass plates were prepared by cutting fresh microscope slides (Corning) into $1 \times 1 \mathrm{~cm}$ pieces.

\section{Instrumentation}

Raman spectra were recorded with a Renishaw System 1000 Raman spectrometer equipped with an integral microscope (Leica DMLMS/N). Radiation of $632.8 \mathrm{~nm}$ from a $25 \mathrm{~mW}$ aircooled He-Ne laser (Renishaw) was used for excitation. Raman scattering was collected with a 50×, $0.75 \mathrm{NA}$ dry objective in $180^{\circ}$ configuration and focused into a Peltier cooled CCD camera $(400 \times 600$ pixels $)$. With a holographic grating (1800 grooves $\mathrm{mm}^{-1}$ ) and a $50 \mu \mathrm{m}$ slit, a spectral resolution of $1 \mathrm{~cm}^{-1}$ can be obtained. A silicon wafer with a Raman band at $520 \mathrm{~cm}^{-1}$ was used to calibrate the spectrometer and the accuracy of the spectral measurement was estimated to be better than $1 \mathrm{~cm}^{-1}$. Scanning transmission electron microscopy (STEM) investigations were performed using a HD-2000 scanning transmission electron microscope (probe size $\approx 0.8 \mathrm{~nm}$ ) operating at $200 \mathrm{kV}$.

\section{Substrate preparation}

Silica beads were synthesized by base-catalyzed hydrolysis of TEOS with the method described by Stöber and Fink. ${ }^{19}$ The compositions for synthesizing silica beads with different sizes are listed in Table 1. Briefly, an appropriate mixture of ethanol, ammonia solution, TEOS and deionized water (see Table 1) was stirred overnight at room temperature. The resulting silica bead were then washed and centrifuged several times in ethanol. Subsequently, the silica bead suspension in ethanol was deposited on a clean, glass microscope slide. After evaporation of the ethanol, a film of silica beads was obtained. The film was then heated at $550^{\circ} \mathrm{C}$ for $2 \mathrm{~h}$ to enhance adhesion of the silica beads to the glass slide.

Silver (wire, 99.9\%; Johnson Matthey) was evaporated on to the silica bead film by using a laboratory-built vacuum evaporator. The evaporator essentially consisted of a tungsten filament wrapped with silver wire. The glass slide with the silica bead film was mounted on a stainless steel disk and placed directly below the filament. No collimating aperture separated the sample surface from the filament. The deposition was done with a constant filament current of 9 A. The mass thickness of the silver film was measured with a quartz crystal microbalance (QCM) placed at the same height as the glass slide. A $6 \mathrm{MHz}$ AT-cut quartz crystal (12.5 $\mathrm{mm}$ in

Table 1. Composition used in preparation of silica beads with different sizes

\begin{tabular}{|c|c|c|c|c|}
\hline Ethanol/ml & $\begin{array}{l}\text { Ammonia } \\
\text { solution/g }\end{array}$ & $\begin{array}{l}\text { Tetraethyl } \\
\text { orthosili- } \\
\text { cate/g }\end{array}$ & Water/g & $\begin{array}{c}\text { Average } \\
\text { size of silica } \\
\text { beads } / \mathrm{nm}\end{array}$ \\
\hline 150 & 12.58 & 9.36 & 0 & 128 \\
\hline 150 & 4.55 & 7.81 & 16.97 & 161 \\
\hline 150 & 18.70 & 9.36 & 0 & 435 \\
\hline 150 & 25.00 & 9.36 & 0 & 565 \\
\hline 150 & 37.50 & 9.36 & 0 & 800 \\
\hline
\end{tabular}


diameter) with gold electrodes (6 $\mathrm{mm}$ in diameter) on each side was used as the QCM resonator. The QCM was driven with an ICM Lever Oscillator (Model 35366). Data were acquired with an Agilent 53131A Universal Counter. The pressure of the system during deposition was $\sim 2 \times 10^{-6}$ Torr $(1$ Torr $=133.3 \mathrm{~Pa})$. For SERS characterization, $10 \mu \mathrm{l}$ of a methanol solution containing a known concentration of benzoic acid were applied on to the SERS substrate. Raman measurements were performed after evaporation of methanol. ${ }^{12}$

\section{RESULTS AND DISCUSSION}

\section{Silver on silica bead films}

Figure 1 shows an SEM image of a typical thin film formed by silica beads. After deposition of the silver on the silica beads, SEM images were obtained in order to examine the morphology of the silver. As shown in Fig. 2, nanometer-sized silver islands, or substructures, covered

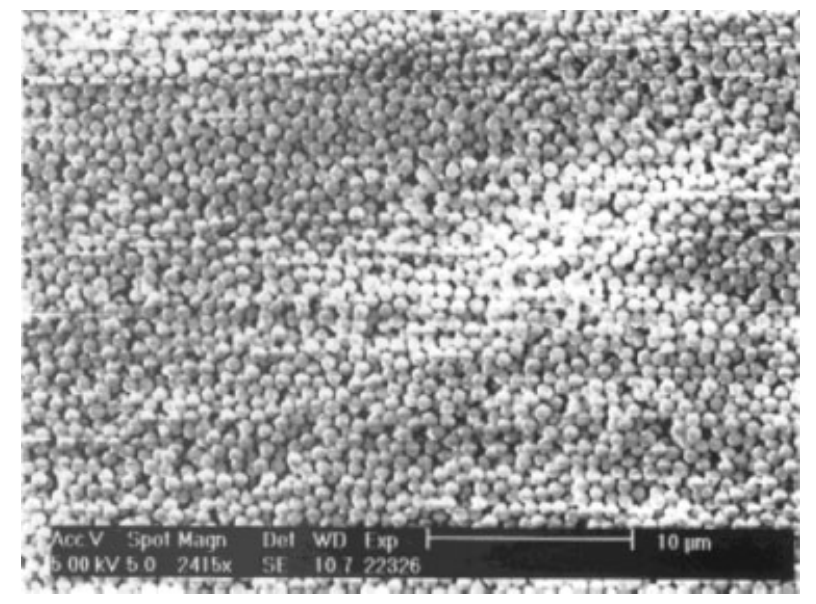

Figure 1. Scanning electron micrograph of film formed by silica beads.

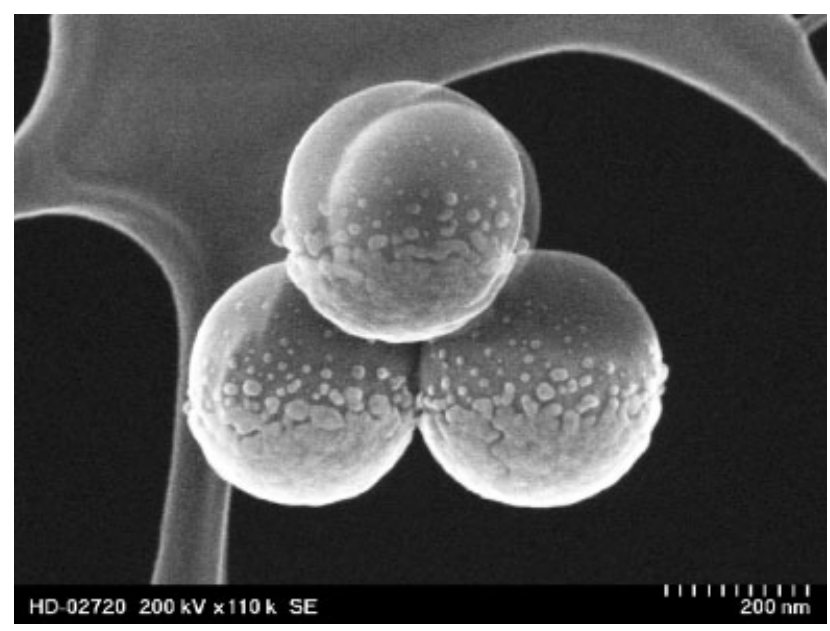

Figure 2. Scanning electron micrograph of $\mathrm{Ag} / \mathrm{SiO}_{2}$ bead substrate. The silica bead is $350 \mathrm{~nm}$ in diameter. the top of the silica beads. These silver substructures are primarily responsible for the enhancement of the Raman signal. As mentioned by Litorja et al., the substructure topography of the silver film is essentially dependent only on the metal evaporation rate. ${ }^{17}$ Therefore, films that are deposited at equivalent evaporation rates are expected to exhibit similar substructure topographies and produce comparable signal enhancements.

\section{Influence of Ag thickness on SERS response}

The effect of the mass thickness of the silver film on the SERS response was studied using the $1003 \mathrm{~cm}^{-1}$ peak of benzoic acid. Figure 3 shows that the sensitivity of the substrate to adsorbed benzoic acid improved with longer deposition time until a maximum value of signal enhancement was reached. Further increase of the film thickness resulted in a decline in the sensitivity. Scanning electron micrographs of the substrate at various points in the deposition process were taken to compare the surface topology of the film at different thickness values. Figure 4(a)-(d) shows SEM images of silver-coated silica beads corresponding to silver deposition times of 4, 32, 47 and 67 minutes, respectively. At short deposition times, a small number of silver islands with diameters of a few nanometers appeared on the silica bead surface. Not surprisingly, more of these small silver islands developed as the mass thickness increased. Continued deposition resulted in the emergence of larger silver islands in the size range of tens of nanometers. Thus, one possible explanation of the peak observed in the SERS response is that as the thickness increased, the number of larger silver islands increased. Since these islands are in the size range for optimal SERS enhancement, the response improved. When the thickness was further increased, these larger silver substructures were somewhat covered resulting in the observed decrease in SERS signal. A deposition time of $47 \mathrm{~min}$ produced the film with maximum response. From

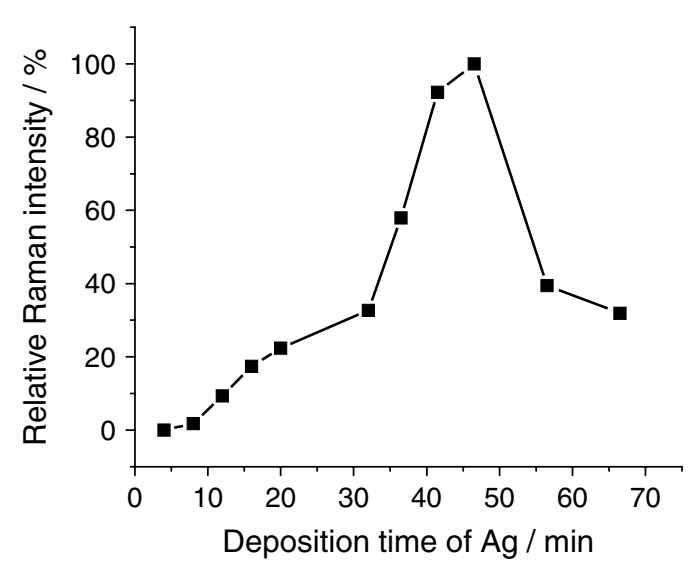

Figure 3. Influence of mass thickness of Ag film on SERS response. The diameter of the silica bead is $650 \mathrm{~nm}$ and the benzoic acid concentration tested is $10^{-2} \mathrm{M}$. 
(a)

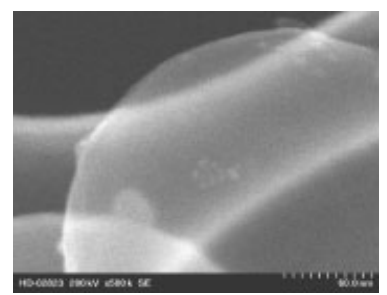

(c)

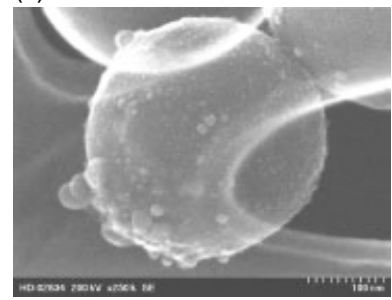

(b)

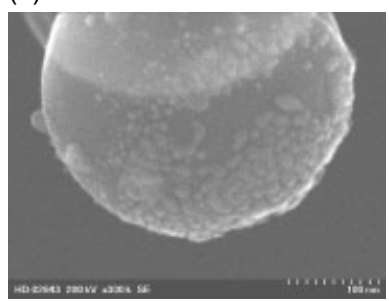

(d)

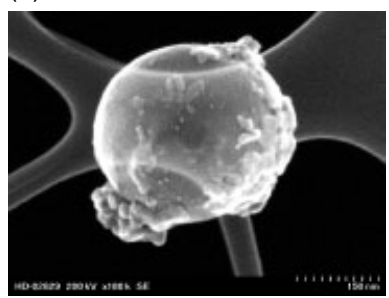

Figure 4. Scanning electron micrographs of the $\mathrm{Ag} / \mathrm{SiO}_{2}$ beads substrate during deposition process. The silica bead is $350 \mathrm{~nm}$ in diameter. The deposition times in (a), (b), (c) and (d) are 4, 32, 47 and $67 \mathrm{~min}$, respectively.

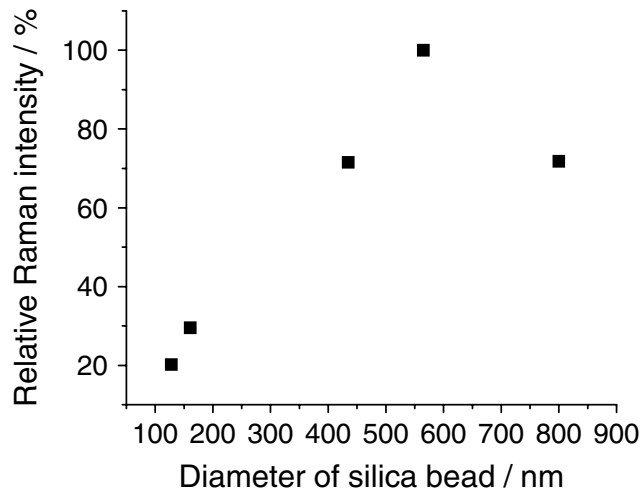

Figure 5. Influence of diameter of silica bead on SERS response. The mass thickness of $\mathrm{Ag}$ is about $4 \mathrm{~nm}$.

QCM measurements, the average mass thickness was $\sim 6 \mathrm{~nm}$ for the optimized substrate.

\section{Influence of the silica bead dimensions on SERS response}

The dimensions of the silica beads used to form the substrates were found to influence the SERS response even when coated with an identical silver film thickness. Figure 5 shows the response as a function of silica bead diameter. The films made with $565 \mathrm{~nm}$ silica beads yielded the maximum response. SEM images were taken to explore the relationship between bead diameter and silver morphology. The micrographs are shown in Fig. 6. It is important to note that the topography of the silver is similar for the various bead diameters even though the Raman signal of benzoic acid is different. This similarity is expected since the morphology of the silver substructures depends only on the silver evaporation rate (a)

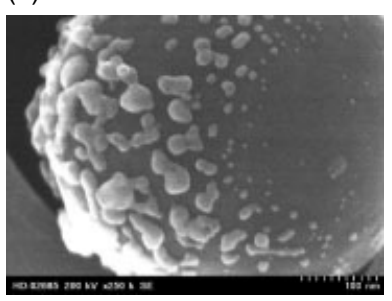

(b)

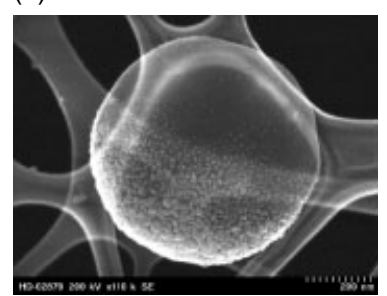

(c)

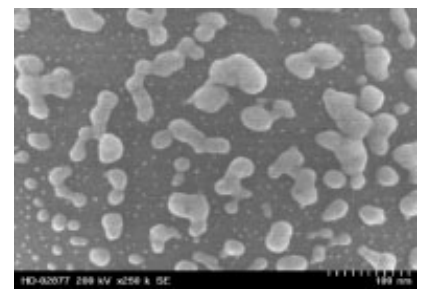

Figure 6. Scanning electron micrographs of the Ag over silica beads with different diameters: (a) 435; (b) 650; (c) $800 \mathrm{~nm}$. and not on the size of the substrate. Experiments are in progress to investigate possible reasons for this dependence of the SERS signal on the size of the silica beads.

\section{Calibration curve for benzoic acid detection}

Figure 7 shows a typical spectrum of benzoic acid $(5 \times$ $10^{-4} \mathrm{M}$ ) adsorbed on the silver film over the silica bead. Despite the high background, the peaks at 1003 and $1600 \mathrm{~cm}^{-1}$ could be readily distinguished. The quantitative performance of the silver film over silica bead surface was evaluated by depositing samples of benzoic acid solution of increasing concentration (from $5 \times 10^{-7}$ to $10^{-1} \mathrm{M}$ ) on the substrate. The logarithmic calibration graph is shown in Fig. 8. The data were obtained with the most intense peak

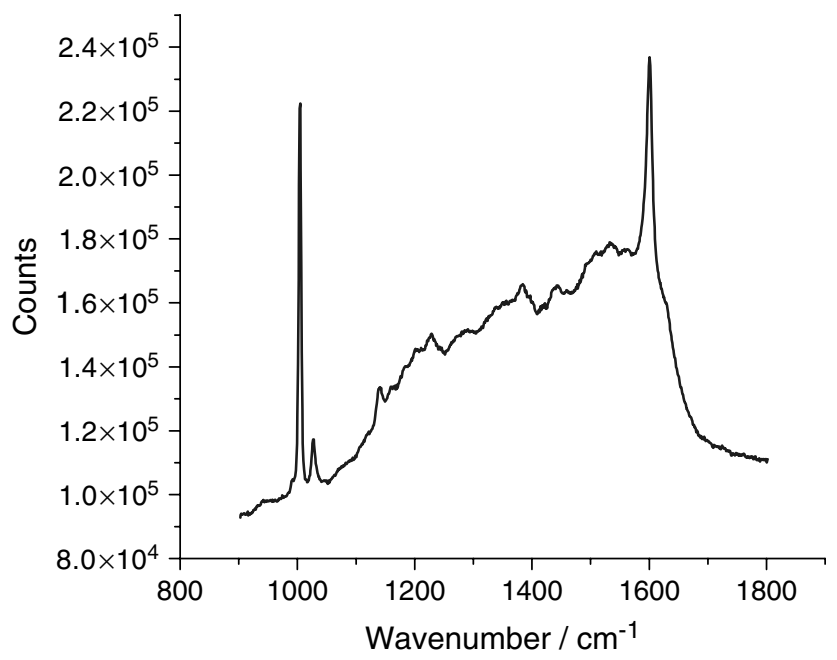

Figure 7. SERS spectrum of benzoic acid $\left(5 \times 10^{-4} \mathrm{M}\right)$ adsorbed on $\mathrm{Ag} / \mathrm{SiO}_{2}$ bead substrate. The silica bead is $650 \mathrm{~nm}$ in diameter and the mass thickness of $\mathrm{Ag}$ is about $6 \mathrm{~nm}$. 


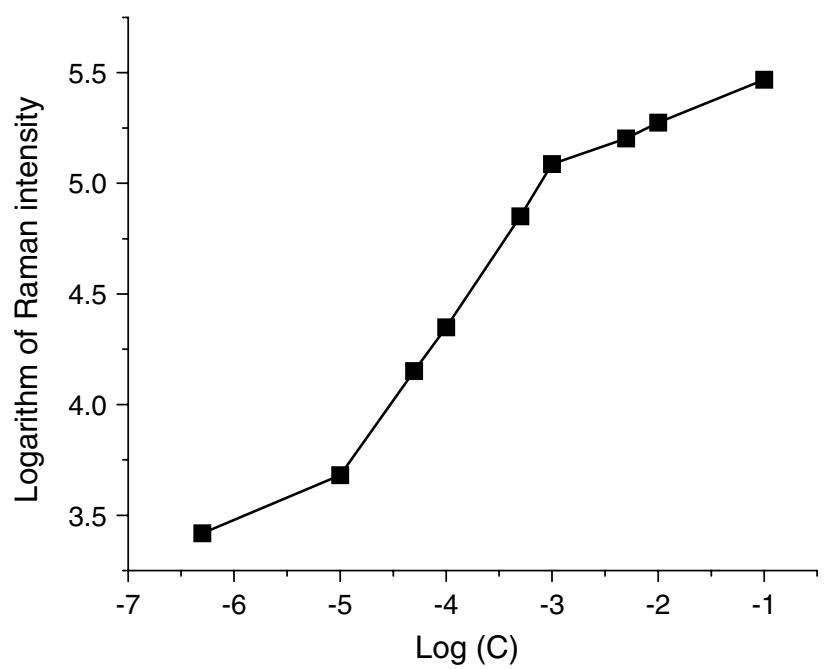

Figure 8. Calibration graph of benzoic acid adsorbed on $\mathrm{Ag} / \mathrm{SiO}_{2}$ beads. The silica bead is $650 \mathrm{~nm}$ in diameter and the mass thickness of $\mathrm{Ag}$ is about $6 \mathrm{~nm}$.

at $1003 \mathrm{~cm}^{-1}$, using $632.8 \mathrm{~nm}$ laser radiation excitation with a laser power of $25 \mathrm{~mW}$. The detection limit obtained was $5 \times 10^{-7} \mathrm{M}$. The relative standard deviation for three tests was $5 \%$. In the ranges $10^{-5}-10^{-3}$ and $10^{-3}-10^{-1} \mathrm{M}$, the SERS signal was linearly related to the concentration of benzoic acid with two different slopes. Benzoic acid concentrations higher than $10^{-1} \mathrm{M}$ damaged the film and are not included in the analysis.

\section{CONCLUSIONS}

Films made by vacuum depositing silver on silica nanospheres were investigated as SERS substrates. Scanning electron micrographs of the substrates were taken to probe the effect of silver morphology on the SERS response. In particular, growth of nanometer size silver substructures, or islands, appears to be primarily responsible for the signal enhancement. The sensitivity of this particular substrate was also significantly influenced by the size of the silica beads. A film made by evaporating a $6 \mathrm{~nm}$ layer of silver on to $565 \mathrm{~nm}$ silica beads exhibited the best sensitivity to adsorbed benzoic acid with a measured detection limit of $5 \times 10^{-7} \mathrm{M}$. In the two ranges $10^{-5}-10^{-3}$ and $10^{-3}-10^{-1} \mathrm{M}$, the SERS signal was linearly related to the concentration of benzoic acid.

\section{Acknowledgments}

This work was conducted at the Oak Ridge National Laboratory and supported by the Environmental Management Science Program (EMSP), US Department of Energy, under contract No. DE-AC0500OR22725 with UT-Battelle, LLC. This research was supported in part by the appointments for L-L.B. and S.M.M. to the ORNL Research Associates Program, administered jointly by ORNL and the Oak Ridge Institute for Science and Education. We thank Dr Byunghwan Lee, Dr Zheng-Wei Pan and Dr Zong-Tao Zhang for SEM measurements.

\section{REFERENCES}

1. Chang RK, Furtak TE. Surface Enhanced Raman Scattering. Plenum Press: New York, 1982.

2. Fleischmann M, Hendra PJ, McQuillan AJ. Chem. Phys. Lett. 1974; 26: 163.

3. Lasema JJ, Torres EL, Winefordner JD. Anal. Chim. Acta 1987; 200: 469.

4. Freeman RG, Grabar KC, Allison KJ, Bright RM, Davis JA, Guthrie AP, Hommer MB, Jackson MA, Smith PC, Walter DG, Natan MJ. Science 1995; 267: 1629.

5. Semin DJ, Rowlen KL. Anal. Chem. 1994; 66: 4324.

6. Van Duyne RP, Hulteen JC, Treichel DA. J. Chem. Phys. 1993; 99: 2101.

7. Schlegel VL, Cotton TM. Anal. Chem. 1991; 63: 241.

8. Moody RL, Vo-Dinh T, Fletcher WH. Appl. Spectrosc. 1987; 41: 966.

9. Ni F, Cotton TM. Anal. Chem. 1986; 58: 3159.

10. Miller S, Baiker A, Meier M, Wokaun A. J. Chem. Soc., Faraday Trans. I 1984; 80: 1305.

11. Dai S, Lee YH, Young JP. Appl. Spectrosc. 1996; 50: 536.

12. Lee YH, Dai S, Young JP. J. Raman Spectrosc. 1997; 28: 635.

13. Liu FX, Xia Y, Li YS. J. Raman Spectrosc. 2001; 32: 73.

14. Muniz-Miranda M. J. Raman Spectrosc. 2002; 33: 295.

15. Compagnini G, D’ Urso L, Puglisi O. Mater. Sci. Eng. C 2002; 19: 295.

16. Kobayashi Y, Salgueirino-Maceira V, Liz-Marzan LM. Chem. Mater. 2001; 13: 1630.

17. Litorja M, Haynes CL, Haes AJ, Tensen TR, Van Duyne RP. J. Phys. Chem. B 2001; 105: 6907.

18. Dick LA, McFarland AD, Haynes CL, Van Duyne RP. J. Phys. Chem. B 2002; 106: 853.

19. Stöber W, Fink A. J. Colloid Interface Sci. 1968; 26: 62. 